\title{
EVALUATION CODES AND PLANE VALUATIONS
}

\author{
C. GALINDO AND M. SANCHIS
}

\begin{abstract}
We apply tools coming from singularity theory, as Hamburger-Noether expansions, and from valuation theory, as generating sequences, to explicitly describe order functions given by valuations of 2-dimensional function fields. We show that these order functions are simple when their ordered domains are isomorphic to the value semigroup algebra of the corresponding valuation. Otherwise, we provide parametric equations to compute them. In the first case, we construct, for each order function, families of error correcting codes which can be decodified by the Berlekamp-Massey-Sakata algorithm and we give bounds for their minimum distance depending on minimal sets of generators for the above value semigroup.
\end{abstract}

\section{INTRODUCTION}

To treat of laying the foundations of algebraic geometry codes, in [8] was introduced the concept of order function, which allows to study, in a unique treatment, classical codes as the duals of one-point geometric Goppa codes or weighted Reed-Muller codes.

Order functions are defined on the so-called order domains and they provide valuation rings that are included in the quotient field of such an order domains. This fact has been established in [9] and used to give a non-usual example of an order function, on a polynomial ring in two indeterminates, which does not correspond to any monomial ordering. Although, that paper supplies many interesting examples, it does not contain a systematic development to describe and compute order functions given by valuations.

Furthermore, in [6], the concept of order function has been enlarged in such a way that the image set of an order function needs not be a subsemigroup of that of the nonnegative integers but only a well-ordered semigroup. With the help of these order functions, by using evaluation maps, error-correcting codes, called evaluation codes, can be constructed. The main achievements of the dual codes of these codes are that bounds for their minimum distance can be given depending on the order function used for their definition and that they can be decodified (in an easy and fast manner) by using the Berlekamp-Massey-Sakata algorithm.

In this paper, we consider valuations of function fields centered at some local ring and show that the semigroup algebras of their value semigroups are ordered domains. We center our study in the 2-dimensional case. We explicitly describe those algebras by regarding them as graded algebras associated with the valuation. This allows to determine parameters for their corresponding evaluation codes. Furthermore, we see that above valuations provide, even over domains different from the semigroup algebra, order functions that can be treated in an algorithmic and very explicit way.

Supported by Spain Ministry of Education MTM2004-00958, GV05/029 and Bancaixa P1-1A2005-08. 
To do it, we give a deep description of the above cited valuations, focused to a simple computation of their value semigroups and to easily handle those order functions that they determine. We consider a refinement of Zariski's classification of these valuations in the line given in [10], but presented in terms of the so-called Hamburger-Noether expansions. These expansions, for curve singularities over algebraically closed fields whose characteristic needs not be zero, were introduced by Campillo in [1] and we can use the computer algebra system SINGULAR [7] to compute them. More explicitly, we consider valuations of the quotient field $K$ of a 2-dimensional Noetherian local regular domain $R$, centered at $R$, called plane valuations and a classification for these ones in terms which allow us to explicitly manipulate them. We also assume that $R$ has an algebraically closed coefficient field $k$ of arbitrary characteristic.

By using the above classification and the concept of generating sequence of a valuation (Definition 4.1), we shall show that for all plane valuations $\nu$ but those of type A and some of type B, $k[S], S$ being the value semigroup of $\nu$, can be regarded as a graded algebra relative either to $\nu$ and $R$ or to $o:=-\nu$ and to a subring of $K$. (Note that when the valuation is of type A or B-I, the semigroup $S$ coincides with the semigroup of a germ of irreducible curve and it is a numerical one). The advantages of the above construction are that it allows to determine generators for $S$, the defining ideal of $k[S]$ (that ideal $I$ of some polynomial ring $A$ such that $k[S] \cong A / I)$ and to see how $o$ works over $k[S]$. Furthermore, we can decide when the associated order function (which is of a particular type called weight function) is not of monomial type. Since we can describe this semigroup algebra, bounds for the minimum distance of the associated codes can be provided. As a consequence, we are able to explicitly construct evaluation codes (with information about their parameters) over domains whose quotient field is a function field of transcendence degree two. For more general cases, we also prove in Proposition 6.2 that evaluation codes can be constructed.

Finally, we provide ordered domains included in $K$ whose order function is $o$ and we show how to compute $o(h)$ for any element $h \in K$.

Section 2 of the paper is introductory, it shows that the value semigroups of order functions and valuations satisfy analogous properties, and it also gives conditions to that valuations provide weight functions. The concept of Hamburger-Noether expansion of a valuation, the classification and parametric equations to compute plane valuations (and so, weight functions) are presented in Section 3, while the types of valuations which give rise to weight functions and a subring of $K$ whose graded algebra is isomorphic to $k[S]$ are provided in Section 4. Section 5 describes the value semigroup of a plane valuation, the defining ideal of $k[S]$, and how to use this information to give bounds for the minimum distance of the codes associated with $k[S]$. In Section 6 , we explain how to compute order functions and their ordered domains with a unique input: the Hamburger-Noether expansion of a valuation. In some cases we use other existing algorithms for treating curve singularities. We conclude it by giving several examples.

\section{Weight FUnCTIONS AND VALUATIONS}

First at all, we give some definitions for semigroups. Denote by $\alpha, \beta, \gamma$ arbitrary elements in a commutative semigroup $\Gamma$ with zero. Then $\Gamma$ is called cancellative if $\alpha+\beta=\alpha+\gamma$ 
implies $\beta=\gamma$. An order $\leq$ on $\Gamma$ is said to be admissible if, whenever $0 \leq \alpha$ it holds that $\alpha+\gamma \leq \beta+\gamma$ whenever $\alpha \leq \beta$.

Throughout this paper, unless otherwise stated, $\Gamma$ will denote a cancellative well-ordered commutative with zero semigroup, where the order is admissible. Let $\Gamma$ be as above and denote by $\Gamma_{-\infty}$ the semigroup $\Gamma \cup\{-\infty\}$, which is ordered as $\Gamma$ and $-\infty$ is a minimal element. Denote by $k$ an algebraically closed field of arbitrary characteristic, $k^{*}=k \backslash\{0\}$ and by $T$ a $k$-algebra.

Definition 2.1. An order function on $T$ is a mapping $o$ from $T$ onto $\Gamma_{-\infty}$ such that for $f, g \in T$, it must be satisfied the following statements:

- $o(f)=-\infty$ iff $f=0$;

- $o(a f)=o(f)$ for all nonzero element $a \in k^{*}$;

- $o(f+g) \leq \max \{o(f), o(g)\}$;

- If $o(f)=o(g)$, then there exists a nonzero element $a \in k^{*}$ such that $o(f-a g)<$ $o(g)$.

An order function such that it also satisfies $o(f g)=o(f)+o(g)$ is called a weight function.

Definition 2.2. A valuation of a field $K$ is a mapping

$$
\nu: K^{*}(:=K \backslash\{0\}) \rightarrow G,
$$

where $G$ is a totally ordered group such that it satisfies

- $\nu(u+v) \geq \min \{\nu(u), \nu(v)\}$;

- $\nu(u v)=\nu(u)+\nu(v)$

for $u, v \in K^{*}$.

Let $\nu$ a valuation of $K$. The subring of $K, R_{\nu}:=\left\{u \in K^{*} \mid \nu(u) \geq 0\right\} \cup\{0\}$ is called the valuation ring of $\nu . R_{\nu}$ is a local ring whose maximal ideal is $m_{\nu}:=\left\{u \in K^{*} \mid \nu(u)>\right.$ $0\} \cup\{0\}$. We shall call the rank of the valuation $\nu(\operatorname{rk}(\nu))$ the Krull dimension of the ring $R_{\nu}$.

From now on, we shall assume that $(R, m)$ is a Noetherian local regular domain. We say that a valuation $\nu$ of the quotient field of $R$, which in the sequel will be denoted by $K$, is centered at $R$ if $R \subseteq R_{\nu}$ and $R \cap m_{\nu}=m$. In this case, the ideals which are contractions to $R$ of ideals in $R_{\nu}$ are called valuation ideals or $\nu$-ideals. Finally, the subset of $G, \nu(R \backslash\{0\})$, is called the semigroup of the valuation $\nu$ (relative to $R$ ).

Proposition 2.1. The value semigroup $S$ of a valuation $\nu$ of a field $K$, centered at $R$, is a cancellative, commutative, free of torsion, well-ordered semigroup with zero, where the associated order is admissible. Moreover, $F=\left\{P_{\alpha}\right\}_{\alpha \in S}$, where

$$
P_{\alpha}:=\{f \in R \backslash\{0\} \mid \nu(f) \geq \alpha\} \cup\{0\}
$$

is the family of $\nu$-ideals (in $R$ ) of the valuation $\nu$.

Proof. We shall prove that $S$ is free of torsion, $F$ is the family of $\nu$-ideals and, finally, that $S$ is well-ordered. The remaining properties are clear.

Assume that $\nu(u) \neq 0, u \in K \backslash\{0\}$, then either $\nu(u)>0$ or $\nu\left(u^{-1}\right)>0$, so either $u \in m_{\nu}$ or $u^{-1} \in m_{\nu}$ and therefore either $u^{p} \in m_{\nu}$ or $u^{-p} \in m_{\nu}, p$ being a positive integer. Thus $\nu\left(u^{p}\right) \neq 0$ and the group spanned by $S, G(S)$ (which is that satisfying that 
there exists a semigroup homomorphism $\eta: S \rightarrow G(S)$ such that if $H$ is a commutative group and $\xi: S \rightarrow H$ a semigroup homomorphism, then there exists a unique semigroup homomorphism $g: G(S) \rightarrow H$ such that $g \circ \eta=\xi$ ) is free of torsion. This proves that $S$ is also.

$R$ is a Noetherian ring and then $\operatorname{rk}(\nu)<\infty$ (see [12, App. 2]). So, each $\nu$-ideal $I$ is finitely generated. Consider a finite set of generators for $I$ and set $\alpha$ the minimum of the values (by $\nu$ ) of these generators, then it is straightforward that $I=P_{\alpha}$ and so $I \in F$.

Finally, $S$ is well-ordered because the family of $\nu$-ideals $F$ is also [12, App. 3].

We have just proved that the value semigroup relative to a valuation satisfies the same properties as those relative to order functions. Note that the fact that $S$ is free of torsion can also be deduced from the fact that $S$ has an admissible and total well-order.

The following result shows how to get ordered domains from certain valuations.

Proposition 2.2. Let $K$ be the quotient field of a Noetherian local regular domain $R$. Let $\nu: K^{*} \rightarrow G$ be a valuation of $K$ which is centered at $R$ and denote by $S$ its value semigroup. Also assume that the canonical embedding of the field $k:=R / m$ into the field $K_{\nu}:=R_{\nu} / m_{\nu}$ is an isomorphism.

Denote by o the mapping $o: K^{*} \rightarrow G$ given by $o(u)=-\nu(u)$ and let $A \subseteq K^{*}$ be a $k$-algebra satisfying that $o(A)$ is a cancellative, commutative, free of torsion, well-ordered semigroup with zero, $\Gamma$, where the associated order is admissible. Then, $o: A \rightarrow o(A)_{-\infty}$, $o(0)=-\infty$, is a weight function.

Proof. We only need to show the last condition defining order functions, since the remaining ones are clear. Firstly, pick $\alpha \in S$ and consider its associated $\nu$-ideal (relative to $R) P_{\alpha}$. Set $P_{\alpha^{+}}:=\{f \in R \mid \nu(f)>\alpha\} \cup\{0\}$, then the $k$-vector space $P_{\alpha} / P_{\alpha^{+}}$is one-dimensional since $k \cong k_{\nu}$.

Consider $f, g \in A(f \neq 0, g \neq 0)$ such that $o(f)=o(g)$. Write $f=u_{1} / v_{1}$ and $g=u_{2} / v_{2}$, where $u_{i}, v_{i} \in R(i=1,2)$. $o(f)=o(g)$ implies $\nu\left(u_{1} v_{2}\right)=\nu\left(u_{2} v_{1}\right)$, we denote by $\alpha \in S$ this value. The cosets of $u_{1} v_{2}$ and $u_{2} v_{1}$ in $P_{\alpha} / P_{\alpha^{+}}$are linearly dependent and thus there exists $\delta \in k$ such that

$$
\nu\left(u_{1} v_{2}-\delta u_{2} v_{1}\right)>\nu\left(u_{1} v_{2}\right) .
$$

So $\nu(f-\delta g)=\nu\left(u_{1} v_{2}-\delta u_{2} v_{1}\right)-\nu\left(u_{1} v_{2}\right)>\nu\left(u_{1} v_{2}\right)-\nu\left(v_{1} v_{2}\right)=\nu\left(u_{1}\right)-\nu\left(v_{1}\right)$, which concludes the proof.

In the rest of this paper, we only consider valuations of the quotient field $K$ of a Noetherian local domain of dimension two $R$, centered at $R$, which we shall call plane valuations. We shall introduce a suitable way to compute them. This procedure will allow us to classify valuations and explicitly compute their value semigroups $S$.

\section{The 2-Dimensional CASE}

3.1. Preliminaries. Valuations were introduced by Krull and they have been studied to treat the desingularization problem in Algebraic Geometry. Zariski in [11] classified plane valuations by attending classical invariants for them as the rank (which is the Krull dimension of their valuation ring) or the rational rank (which is the dimension of the 
$\mathbb{Q}$-vector space $G \otimes_{\mathbb{Z}} \mathbb{Q}, G$ being the value group of the valuation and $\mathbb{Z}$ ( $\mathbb{Q}$, respectively) the set of integer (rational, respectively) numbers).

By using previous results by Zariski, Spivakovsky in [10] gives the following geometrical view of plane valuations.

Theorem 3.1. There is a one to one correspondence between the set of plane valuations (of $K$ centered at $R$ ) and the set of simple sequences of quadratic transformations of the scheme Spec $R$.

Recall that a quadratic transformation of a 2-dimensional scheme $X$ consists of blowing it up at a closed point $P$ which means, essentially, replacing the point $P$ by a projective line called the exceptional divisor. The correspondence in Theorem 3.1 works as follows: each valuation $\nu$ is associated with the sequence

$$
\pi: \cdots X_{N+1} \stackrel{\pi_{N+1}}{\longrightarrow} X_{N} \longrightarrow \cdots \longrightarrow X_{1} \stackrel{\pi_{1}}{\longrightarrow} X_{0}=X=\operatorname{Spec} R,
$$

where $\pi_{i+1}$ is the blowing-up of $X_{i}$ at the unique closed point $P_{i}$ of the exceptional divisor $L_{i}$ (that obtained after the blowing-up $\pi_{i}$ ) satisfying that $\nu$ is centered at the local ring $\mathcal{O}_{X_{i}, P_{i}}\left(:=R_{i}\right)$.

Theorem 3.1 allows Spivakovsky to give a classification of plane valuations which improves Zariski's and it is based in the form of the so-called dual graph of the sequence $\pi$. Note that this graph reflects the relative position of the exceptional divisors of $\pi$. Moreover, he notices that the behaviour of plane valuations is similar to that of germs of plane curves.

However, the dual graph is not useful when we want to get parametric equations for computing valuations. Furthermore, the classical theory for curves uses, for this purpose, Puiseux exponents that only work for zero characteristic.

We take an interest in coding theory and, so, we are interested in positive characteristic. Therefore, we are going to give a classification (which is basically the one given in [10]) but expressed in terms of the so-called Hamburger-Noether expansions. These expansions have been used in [5] to study saturation with respect to valuations of 2-dimensional Noetherian local regular domains.

3.2. Hamburger-Noether expansions and classification of plane valuations. Let $\nu$ be a plane valuation (of $K$ centered at $R$ ) and take $\{u, v\}$ a regular system of parameters for the ring $R$. Assume that $\nu(u) \leq \nu(v)$. This means that there exists an element $a_{01} \in k$ such that the set $\left\{u_{1}=u, v_{1}=(v / u)-a_{01}\right\}$ constitutes a regular system of parameters for the ring $R_{1}$. If, now, $\nu(u) \leq \nu\left(v_{1}\right)$ holds, then we repeat the above operation and we keep doing the same thing until we get

$$
v=a_{01} u+a_{02} u^{2}+\cdots+a_{0 h} u^{h}+u^{h} v_{h},
$$

where either $\nu(u)>\nu\left(v_{h}\right)$ or $\nu\left(v_{h}\right)=0$, or

$$
v=a_{01} u+a_{02} u^{2}+\cdots+a_{0 h} u^{h}+\cdots
$$

with infinitely many steps.

In the last two cases, we have got the Hamburger-Noether expansion for $\nu$, obtaining $R_{\nu}=R_{h}$ when $\nu\left(v_{h}\right)=0$. Otherwise, set $w_{1}:=v_{h}$ and reproduce the above procedure for 
the regular system of parameters $\left\{w_{1}, u\right\}$ of $R_{h}$. As a consequence, we obtain an ordered family of equalities which have the form

$$
w_{j-1}=\sum_{i=1}^{h_{j}} a_{j i} w_{j}^{i}+w_{j}^{h_{j}} w_{j+1} .
$$

The procedure could continue indefinitely or we could obtain a last equality like (2) whose index $j$ will be denoted by $z$. By simplicity's sake, we write $z \leq \infty$, where $z=\infty$ means that there is no last parameter $w$. Therefore, we can associate to each plane valuation $\nu$ a set of expressions, depending on a regular system of parameters $\{u, v\}$ of $R$, which provides a regular system of parameters for each local ring $R_{i}$ given by the sequence $\pi$ described in Section 3.1.

This set of equations is called the Hamburger-Noether expansion of the valuation $\nu$ in the regular system of parameters $\{u, v\}$ of the ring $R$ and it has the form

$$
\begin{aligned}
v= & a_{01} u+a_{02} u^{2}+\cdots+a_{0 h_{0}} u^{h_{0}}+u^{h_{0}} w_{1} \\
u= & w_{1}^{h_{1}} w_{2} \\
\vdots & \vdots \\
w_{s_{1}-2}= & w_{s_{1}-1}^{h_{s_{1}-1}} w_{s_{1}} \\
w_{s_{1}-1}= & a_{s_{1} k_{1}} w_{s_{1}}^{k_{1}}+\cdots+a_{s_{1} h_{s_{1}}} w_{s_{1}}^{h_{s_{1}}}+w_{s_{1}}^{h_{s_{1}}} w_{s_{1}+1} \\
\vdots & \vdots \\
w_{s_{g}-1}= & a_{s_{g} k_{g}} w_{s_{g}}^{k_{g}}+\cdots+a_{s_{g} h_{s_{g}}} w_{s_{g}}^{h_{s_{g}}}+w_{s_{g}}^{h_{s_{g}}} w_{s_{g}+1} \\
\vdots & \vdots \\
w_{i-1}= & w_{i}^{h_{i}} w_{i+1} \\
\vdots & \\
\left(w_{z-1}=\right. & \left.w_{z}^{\infty}\right) .
\end{aligned}
$$

Notice that the family $\left\{s_{i}\right\}_{i=0}^{g}$ of nonnegative integers is the set of indices corresponding to those rows (called free rows) of the expression (3) which have some nonzero $a_{j l}$. It is clear that $0<s_{1}<s_{2}<\cdots<s_{g} \leq z, g \in \mathbb{N} \cup\{\infty\}$ and $k_{j}=\min \left\{n \in \mathbb{N} \mid a_{s_{j}, n} \neq 0\right\}$, where $\mathbb{N}$ is the set of non-negative integers.

In accordance to its Hamburger-Noether expansion, we classify plane valuations (of $K$ centered at $R$ ) in the following five types.

- Type A.

A plane valuation $\nu$ will be called of type A, whenever its Hamburger-Noether expansion is finite and its last row has the following shape

$$
w_{s_{g}-1}=a_{s_{g} k_{g}} w_{s_{g}}^{k_{g}}+\cdots+a_{s_{g} h_{s_{g}}} w_{s_{g}}^{h_{s_{g}}}+w_{s_{g}}^{h_{s_{g}}} w_{s_{g}+1},
$$

where $w_{s_{g}+1} \in R_{\nu}$ and $\nu\left(w_{s_{g}+1}\right)=0$. Clearly $g<\infty, h_{s_{g}}<\infty$ and $z=s_{g}$.

- Type B. 
We shall say that a plane valuation is of type B when its Hamburger-Noether expansion has a last equality associated with an infinite sum like this

$$
w_{s_{g}-1}=\sum_{j=k_{g}}^{\infty} a_{s_{g} j} w_{s_{g}}^{j} .
$$

It is clear that $g<\infty, h_{s_{g}}=\infty$ and $z=s_{g}$.

- Type C.

A plane valuation is of type $\mathrm{C}$ if its Hamburger-Noether expansion has a last free row like this

and, after, finitely many non-free rows with the shape

$$
w_{s_{g}-1}=a_{s_{g} k_{g}} w_{s_{g}}^{k_{g}}+\cdots+a_{s_{g} h_{s_{g}}} w_{s_{g}}^{h_{s_{g}}}+w_{s_{g}}^{h_{s_{g}}} w_{s_{g}+1}
$$

$$
\begin{array}{ccc}
w_{s_{g}} & = & w_{s_{g}+1}^{h_{s_{g}+1}} w_{s_{g}+2} \\
\vdots & & \vdots \\
w_{z-1} & = & w_{z}^{\infty} .
\end{array}
$$

Here $g<\infty, h_{z}=\infty$ and $s_{g}<z<\infty$.

- Type D.

A plane valuation will be called of type D, whenever its Hamburger-Noether expansion has a last free row like this

$$
w_{s_{g}-1}=a_{s_{g} k_{g}} w_{s_{g}}^{k_{g}}+\cdots+a_{s_{g} h_{s_{g}}} w_{s_{g}}^{h_{s_{g}}}+w_{s_{g}}^{h_{s_{g}}} w_{s_{g}+1}
$$

followed by infinitely many rows with the shape

$$
w_{i-1}=w_{i}^{h_{i}} w_{i+1}
$$

$\left(i>s_{g}\right)$. Clearly, $g<\infty$ and $z=\infty$.

- Type E.

When the Hamburger-Noether expansion of a plane valuation repeats indefinitely the basic structure, then the valuation is called to be of type $E$. That is to say, there exist infinitely many ordered sets of equalities with the shape

$$
\begin{aligned}
w_{s_{i}-1}= & a_{s_{i} k_{i}} w_{s_{i}}^{k_{i}}+\cdots+a_{s_{i} h_{s_{i}}} w_{s_{i}}^{h_{s_{i}}}+w_{s_{i}}^{h_{s_{i}}} w_{s_{i}+1} \\
\vdots & \vdots \\
w_{s_{i+1}-2}= & w_{s_{i+1}-1}^{h_{s_{i+1}-1}} w_{s_{i+1}} .
\end{aligned}
$$

Here $g=z=\infty$.

Notice that this classification does not depend on the regular system of parameters we choose on $R$.

Table 1 relates our classification to that given by Zariski. We have added a new invariant, the transcendence degree (in short tr.deg) of $K_{\nu}$ over $k$, which is important for us as Proposition 2.2 shows.

Remark. As the table shows, classical invariants provide a refinement of type B valuations. In the course of the paper, we shall clarify the reason for it. We are not interested in type 


\begin{tabular}{||c|c|c|c|c||}
\hline type & subtype & rk & rat. rk & tr. deg \\
\hline \hline A & - & 1 & 1 & 1 \\
\hline B & I & 2 & 2 & 0 \\
& II & 1 & 1 & 0 \\
\hline C & - & 2 & 2 & 0 \\
\hline D & - & 1 & 2 & 0 \\
\hline E & - & 1 & 1 & 0 \\
\hline
\end{tabular}

TABLE 1. Invariants and classification of plane valuations.

A valuations since its value semigroup is that of a germ of irreducible curve and because Table 1 shows that its transcendence degree is 1 , however for the sake of completeness and, essentially, because all plane valuations can be regarded as a limit of type A valuations, we also consider them. The idea, to understand this, is that each divisor appearing in the sequence $\pi$ associated with a plane valuation $\nu$, defines a type A valuation $\nu_{i}$ centered at $R$. By bearing in mind that $R_{\nu}$ is the directed limit of the sequence of rings $R_{i}$, it can be proved that the valuation $\nu$ and the so-called limit valuation of the valuations $\nu_{i}$, $\lim _{i \rightarrow \infty} \nu_{i}$, are equivalent and so analogous for our purposes. Notice that no valuation of type A satisfies that the dimension of the $k$-vector spaces $P_{\alpha} / P_{\alpha^{+}}$equals one for all elements $\alpha$ in the value semigroup of $\nu$.

3.3. Parametric equations of a plane valuation. In this Section, we show, case by case, how to obtain parametric equations for any plane valuation $\nu$ (of $K$ centered at $R$ ). These equations depend on one or two parameters according the type of valuation. Moreover, we also explain how to compute the value $\nu(h)$ for any $h \in R$. It is obvious that this allows us to compute $\nu(h)$ for any $h \in K$ and so $o(h), o$ being the above defined weight function. Note that we make our computations in some normalization of $\nu$ (or $o$ ). Other normalizations give rise to equivalent valuations. The associated order functions and their related codes do not depend on the normalization we have chosen.

- Type A.

Let us assume that $\nu$ is a type A plane valuation and that $X_{N+1} \rightarrow X_{N}$ is the last blowing-up (centered at $P_{N}$ ) of its associated sequence $\pi$. Consider its Hamburger-Noether expansion $E$ and set $w_{s_{g}}=t_{1}$ and $w_{s_{g}+1}=t_{2}$. By performing back substitution on $E$, we get parametric equations for $\nu$, which we shall write $u=u\left(t_{1}, t_{2}\right), v=v\left(t_{1}, t_{2}\right)$. It is clear that both expressions are polynomials in the indeterminates $t_{1}$ and $t_{2}$.

Recall Section 3.1. If $h_{1}$ and $h_{2}$ are elements in $R$, we shall denote by $I\left(h_{1}, h_{2}\right)$ the intersection multiplicity, at the maximal ideal $m$ of $R$, between the germs of curves on $X$ $=$ Spec $R$ that define $h_{1}$ and $h_{2}$. On the other hand, an analytically irreducible element in $R$ that defines a germ of plane curve whose strict transform in $X_{N}$ is not singular and intersects $L_{N}$ transversely at $P_{N}$ is called to be a general element of the valuation $\nu$ (relative to $R$ ). In [10], it is proved that for any $h \in R$

$$
\nu(h)=\min \{I(h, f) \mid f \text { is a general element of } \nu\} .
$$


As a consequence, from the behaviour of the germs of plane curves (see [1] for instance), we obtain that

$$
\nu(h)=\nu_{t_{1}}\left[h\left(u\left(t_{1}, t_{2}\right), v\left(t_{1}, t_{2}\right)\right)\right],
$$

where $\nu_{t_{1}}$ maps $h\left(u\left(t_{1}, t_{2}\right), v\left(t_{1}, t_{2}\right)\right)$ into the least exponent of the parameter $t_{1}$.

- Type B.

Suppose that $\nu$ is a plane valuation of type B. Let $\hat{R}$ the $m$-adic completion of the ring $R$. Clearly, we can pick an irreducible element $f \in \hat{R}$, which defines an analytically irreducible germ of curve, having (in a suitable basis of $\hat{R} /(f):\{u+(f), v+(f)\})$ the same HamburgerNoether expansion (as a curve) as $\nu$. So, setting $w_{s_{g}}=t$ and performing back substitution in the Hamburger-Noether expansion of $\nu$, we can conclude that $u=u(t), v=v(t)$ are parametric equations for the valuation $\nu$. Both equations are in the ring of formal power series in the indeterminate $t$, which we denote by $K[[t]]$.

Finally, if $h \in R$, then

$$
\nu(h)=\left(a, \nu_{t}\left(h_{1}(u(t), v(t))\right)\right.
$$

whenever $\nu_{t}$ is defined as in the case of type A valuations and $h=f^{a} h_{1}$ is the factorization of $h$ in $\hat{R}$ in such a way that $f$ does not divide $h_{1}$. This happens because $\nu$ is the restriction to $K$ of a valuation $\hat{\nu}$ of the fraction field of $\hat{R}$ (centered at $\hat{R}$ ) which satisfies the equality (4). This last fact can be proved from the above consideration on the limit of type A valuations and since the sum of the transcendence degree and the rank of $\hat{\nu}$ equals the dimension of the ring $\hat{R}$ and, thus, $\hat{\nu}$ is discrete (see [12] and [10]). Notice that a valuation is said to be discrete whenever its value group is discrete, that is it has finite rank and the quotient groups given by the ordered chain of its isolated subgroups are groups of rank 1 .

- Type C.

The shape of the Hamburger-Noether expansion of the valuations $\nu$ of type $\mathrm{C}$ shows that for all nonnegative integer $n$ the inequality $n \nu\left(w_{z}\right)<\nu\left(w_{z-1}\right)$ holds. Moreover, the same reasoning given for type $\mathrm{B}$ valuations proves that $\nu$ is a discrete valuation. As a consequence, in a suitable normalization of the value group $G$ of $\nu$, we get $G=\mathbb{Z}^{2}$ lexicographically ordered, $\nu\left(w_{z}\right)=(0, b)$ and $\nu\left(w_{z-1}\right)=(c, d),(b, c>0)$.

Now, since the local rings $R_{i}$ associated with the sequence $\pi$ relative to $\nu$ constitute an infinite ascending chain satisfying $R_{i}<R_{i+1}$, $<$ being the domination relation (what means that $R_{i} \subset R_{i+1}$ and the contraction of the maximal ideal of $R_{i+1}$ to $R_{i}$ is the maximal ideal of this last ring), we get $R_{\nu}=\cup_{i=z+1}^{\infty} R_{i}$ and so $h \in R \subseteq R_{\nu}$ can be regarded as an element in the ring $k\left[\left[w_{z-1} / w_{z}^{a}, w_{z}\right]\right]$, where $a$ is some positive integer. Then, if

the following equality holds:

$$
h=\sum h_{i j}\left(w_{z-1} / w_{z}^{a}\right)^{i} w_{z}^{j}
$$

$$
\nu(h)=\min \left\{i(c, d-a b)+j(0, b) \mid h_{i j} \neq 0\right\} .
$$

This proves that setting $w_{z-1}=t^{(c, d)}$ and $w_{z}=t^{(0, b)}$, and performing back substitution in the Hamburger-Noether expansion of $\nu$, we get parametric equations for $\nu, u=u(t)$, $v=v(t), u(t)$ and $v(t)$ being in the algebra $k\left[\mathbb{Z}^{2}\right]$ of the group $\mathbb{Z}^{2}$.

Finally, if $\nu_{t}$ is defined as above but over a suitable subset of $k\left[\mathbb{Z}^{2}\right]$ and under the lexicographical order on $\mathbb{Z}^{2}$, then $\nu(h)=\nu_{t}(h(u(t), v(t))$. 
- Type D.

Assume that $\nu$ is a type $D$ valuation. Since $\nu=\lim _{i \rightarrow \infty} \nu_{i}$ with the notations given in the remark at the bottom of the above section, to obtain parametric equations for $\nu, u=u(t)$, $v=v(t)$, we must consider the real but non rational number $\delta$ given by the continued fraction

$$
\left[h_{s_{g}+1} ; h_{s_{g}+2}, h_{s_{g}+3}, \ldots\right],
$$

write $w_{s_{g}+1}=t$ and $w_{s_{g}}=t^{\delta}$ and perform back substitution in the Hamburger-Noether expansion of $\nu$. Notice that both $u(t)$ and $v(t)$ are elements in $k\langle t\rangle, k\langle t\rangle$ being the ring of formal power series $\sum_{r \in \mathbb{R}} a_{r} t^{r}$ such that $a_{r} \in k$ and the set $\left\{r \in \mathbb{R} \mid a_{r} \neq 0\right\}$ is a well-ordered subset of the set of real numbers, $\mathbb{R}$, under the usual ordering.

Finally, it is clear that for the same definition of $\nu_{t}$ as above but over $k\langle t\rangle$, one gets $\nu(h)=\nu_{t}(h(u(t), v(t))$, for $h \in R$.

- Type E.

Assume, lastly, that $\nu$ is a type E valuation and consider the expansion given by all the equalities of the Hamburger-Noether expansion of $\nu$ until the $s_{j}$ th one. In this way, we get the Hamburger-Noether expansion of a type A valuation $\nu_{j}$ and if we delete from this last

expression $w_{s_{j}}^{h_{s_{j}}} w_{s_{j}+1}$, we obtain (for a suitable basis) the Hamburger-Noether expansion of an analytically irreducible germ of curve. Assuming that the characteristic of $k$ is zero, this gives equations $v=\sum a_{j r} u^{r}, a_{j r} \in k$ and $r \in \mathbb{Q}$ (Puiseux expansions) which does not depend on $j$ (see $[1$, Sect. 3]). Taking into account that $\nu$ is a limit of type A valuations including the $\nu_{j}$ ones, we conclude that the above sums give rise to parametric equations $u=u(t)=t$ and $v=v(t)=\sum_{r \in \mathbb{Q}} a_{r} t^{r} \in k\langle t\rangle$, where the set $\left\{r \in \mathbb{Q} \mid a_{r} \neq 0\right\}$ is infinite and if write each element on it as a quotient of relatively prime elements, the sequence of their denominators is not bounded. Thus, $\nu(h)=\nu_{t}(h(u(t), v(t))$, for $h \in R$.

\section{The Semigroup Algebra as AN ORDERED DOMAin}

Let $\nu$ be a plane valuation (of $K$ centered at $R$ ) and $S$ its value semigroup. Assume that $\nu$ is not of type $\mathrm{A}$. In this section, we shall see that the semigroup algebra $K[S]$ is an ordered domain whose order function depends on $\nu$ and we shall describe it. We shall do this by considering an algebra isomorphic to $k[S]$, the graded algebra relative to $\nu$, and by using the concept of generating sequence of a valuation. Notice that the hypothesis of 2-dimensionality of $R$ is not necessary to define these concepts.

Definition 4.1. A sequence $\left\{r_{i}\right\}_{i \in I}$ of elements in the maximal ideal $m$ of $R$ is said to be a generating sequence (relative to $R$ ) of a valuation $\nu$ if, for any element $\alpha \in S, S$ being the value semigroup of $\nu$, the $\nu$-ideal of $R, P_{\alpha}$, is spanned by the set

$$
\left\{\prod_{j \in I_{0} \subseteq I, I_{0}} r_{j}^{a_{j}} \mid a_{j} \in \mathbb{N}, a_{j}>0 \text { and } \sum_{j \in I_{0}} a_{j} \nu\left(r_{j}\right) \geq \alpha\right\} .
$$

Minimal generating sequences of plane valuations are described in [10]. Next, in our language, we say how to get these sequences.

Firstly, assume that (3) is the Hamburger-Noether expansion for $\nu$. Set $q_{0}=u, q_{1}=$ $v$ and, for $1<i \leq z(z \neq \infty)$, consider $q_{i}$ the defining equation of any analytically 
irreducible germ of curve on Spec $R$ whose Hamburger-Noether expansion in the basis $\left\{\bar{u}=u+\left(q_{i}\right), \bar{v}=v+\left(q_{i}\right)\right\}$ of $\hat{R} /\left(q_{i}\right)$ is

$$
\begin{aligned}
\bar{v}= & a_{01} \bar{u}+a_{02} \bar{u}^{2}+\cdots+a_{0 h_{0}} \bar{u}^{h_{0}}+\bar{u}^{h_{0}} \bar{w}_{1} \\
\bar{u}= & \bar{w}_{1}^{h_{1}} \bar{w}_{2} \\
& \vdots \\
\vdots & \vdots \\
\bar{w}_{s_{1}-1}= & a_{s_{1} k_{1}} \bar{w}_{s_{1}}^{k_{1}}+\cdots+a_{s_{1} h_{s_{1}}} \bar{w}_{s_{1}}^{h_{s_{1}}}+\bar{w}_{s_{1}}^{h_{s_{1}}} \bar{w}_{s_{1}+1} \\
\vdots & \vdots \\
\bar{w}_{s_{i-1}-1}= & a_{s_{i-1} k_{i-1}} \bar{w}_{s_{i-1}}^{k_{i-1}}+\cdots+a_{s_{i-1} h_{s_{i-1}}} \bar{w}_{s_{i-1}}^{h_{s_{i-1}}}+\cdots .
\end{aligned}
$$

Then, a minimal generating sequence of $\nu$ can be obtained as follows, according the type of valuation what $\nu$ belongs to.

Not all valuations have minimal generating sequences. Valuations of type B-II which admit them are called of type B-II-a and the remaining ones will be of type B-II-b. To understand this fact, we have to consider an element $q_{g+1}$ which, in general, will be in the $m$-adic completion $\hat{R}$. $q_{g+1}$ will be the element $f$ given in Section 3.3 which allows $\nu$ to be computed.

If $q_{g+1}$, up to multiplication by an unit, belongs to $R$, then we are speaking about a valuation of type B-II-a, and $\left\{q_{i}\right\}_{i=0}^{g+1}$ is a minimal generating sequence of $\nu$.

Otherwise, if $\nu$ is of type B-II-b, this means that there exists an element in $R$ which, in $\hat{R}$, factorizes as a product which contains $q_{g+1}$ as a factor. When this last fact does not happen, $\nu$ is a type B-I valuation. Neither valuations of type B-II-b nor those of type B-I admit minimal generating sequences.

Let $\nu$ be a plane valuation of type $\mathrm{C}$ or D. In both cases $\left\{q_{i}\right\}_{i=0}^{g+1}$ constitute a minimal generating sequence of $\nu$. In the first type of valuations $\nu\left(q_{i}\right)(0 \leq i<g+1)$ are data lying on the line that joins the origin to $\nu\left(q_{0}\right)$, but $\nu\left(q_{g+1}\right)$ does not satisfy this property. With respect to the second type, $\nu\left(q_{i}\right) \in \mathbb{Q}$ whenever $0 \leq i<g+1$, but $\nu\left(q_{g+1}\right) \in \mathbb{R} \backslash \mathbb{Q}$.

Finally, when $\nu$ is a type E valuation, the infinite sequence $\left\{q_{i}\right\}_{0<i}$ is a minimal generating sequence of $\nu$.

The interest of order functions, for using them in coding theory, is that they provide filtrations $\left\{O_{\alpha}\right\}$ ( $\alpha$ is over the value semigroup of the order function) of the domain $T$, which are defined in such a way that the dimension of the quotient vector spaces $O_{\alpha^{+}} / O_{\alpha}$ is one, $\alpha^{+}$being the next element to $\alpha$.

In the valuative case, this structure fits to that of the so-called graded algebra associated with a valuation:

Definition 4.2. Let $\nu$ be a valuation (of the quotient field $K$ of a local ring $R$ and centered at $R$ ) and $S$ its value semigroup (relative to the ring $R$ ). The graded algebra associated with $\nu$ is defined to be the graded $k$-algebra,

$$
\operatorname{gr}_{\nu} R=\bigoplus_{\alpha \in S} \frac{P_{\alpha}}{P_{\alpha^{+}}}
$$

The following proposition relates minimal generating sequences and the graded algebra of a plane valuation. 
Proposition 4.1. Let $\nu$ be a type B-II-a, $C$ or $D$ plane valuation. Then a set $\left\{r_{i}\right\}_{i \in I}$, where $r_{i} \in m$, is a generating sequence of $\nu$ if, and only if, the $k$-algebra $\operatorname{gr}_{\nu} R$ is spanned by the cosets defined by the elements $r_{i}$ in $\operatorname{gr}_{\nu} R$.

Proof. It suffices to suppose that $\bar{r}_{i}=r_{i}+P_{\nu\left(r_{i}\right)}$ generates $\operatorname{gr}_{\nu} R$ and prove that if $\alpha \in S$ then $P_{\alpha}=Q_{\alpha}, Q_{\alpha}$ being the ideal generated by the set given in (5). This is so because the converse statement of the proposition is straightforward.

Clearly $Q_{\alpha} \subseteq P_{\alpha}$. Let $f \in P_{\alpha}$ be such that $\nu(f)=\alpha_{0} \geq \alpha$, then $f \in P_{\alpha_{0}}$ and $f+P_{\alpha_{0}^{+}} \in \operatorname{gr}_{\nu} R$ is a homogeneous element.

Since $f+P_{\alpha_{0}^{+}}=a \prod \bar{r}_{i}^{\gamma_{i}}, a \in k$, for some vector $\gamma$ whose coordinates are non-negative integers $\gamma_{i}$ and if it is infinite, all its coordinates but finitely many vanish, we get that $f-a \prod r_{i}^{\gamma_{i}} \in P_{\alpha_{0}^{+}}$, and so, $f \in Q_{\alpha_{0}}+P_{\alpha_{0}^{+}}$. Therefore $f+f_{0} \in P_{\alpha_{0}^{+}}$, for some $f_{0} \in Q_{\alpha_{0}}$ and in the same way, $f+f_{0} \in Q_{\alpha_{0}}+P_{\alpha_{1}^{+}}$for some $\alpha_{1} \in S, \alpha_{1}>\alpha_{0}$. Iterating, there appears a increasing sequence $\alpha_{0}<\alpha_{1}<\cdots<\alpha_{i}<\cdots$, such that $\alpha_{i} \in S$, and $f \in Q_{\alpha_{0}}+P_{\alpha_{i}^{+}}$for each $i$. As a consequence

$$
f \in \bigcap_{i=0}^{\infty}\left(Q_{\alpha_{0}}+P_{\alpha_{i}^{+}}\right) .
$$

Set $m$ the maximal ideal of the ring $R$, if we prove that

$$
\bigcap_{i=0}^{\infty}\left(Q_{\alpha_{0}}+P_{\alpha_{i}^{+}}\right)=\bigcap_{i=0}^{\infty}\left(Q_{\alpha_{0}}+m^{i}\right),
$$

then the proof can be completed, because by considering the quotient ring $R / Q_{\alpha_{0}}$ and setting $m+Q_{\alpha_{0}}=\bar{m}$, one gets, in this ring, $\bigcap_{i=0}^{\infty}\left(Q_{\alpha_{0}}+m^{i}\right)=\bigcap_{i=0}^{\infty} \bar{m}^{i}=\overline{0}=Q_{\alpha_{0}}$. This concludes the proof since

$$
\bigcap_{i=0}^{\infty}\left(Q_{\alpha_{0}}+P_{\alpha_{i}^{+}}\right)=Q_{\alpha_{0}}
$$

and so $f \in Q_{\alpha_{0}} \subseteq Q_{\alpha}$.

It only remains to show (6). $\nu$ is a plane valuation of type B-II-a, C or D, therefore it admits a finite minimal generating sequence $\left\{q_{i}\right\}_{i \in\{0, \ldots, g+1\}}$. If $f \in P_{\alpha}$ with $\alpha \in S$ then,

$$
f=\sum_{\gamma \in M_{0} \subseteq M} A_{\gamma} \prod q_{i}^{\gamma_{i}}
$$

where $M=\left\{\gamma=\left(\gamma_{0}, \ldots, \gamma_{g+1}\right) \mid \sum_{j=0}^{g+1} \gamma_{j} \nu\left(q_{j}\right) \geq \alpha\right\}$ and $A_{\gamma} \in R$. Write

$$
\mu_{\alpha}=\min \left\{\sum_{j=0}^{g+1} \gamma_{j} \mid\left(\gamma_{0}, \ldots, \gamma_{g+1}\right) \in M\right\} \text {, }
$$

then it is clear that, $f \in m^{\mu_{\alpha}}$, and moreover $\mu_{\alpha^{\prime}}>\mu_{\alpha}$ whenever $\alpha^{\prime}>\alpha$. Thus $\bigcap_{i=0}^{\infty}\left(Q_{\alpha_{0}}+P_{\alpha_{i}^{+}}\right) \subseteq \bigcap_{i=0}^{\infty}\left(Q_{\alpha_{0}}+m^{i}\right)$. This concludes the proof of $(6)$, because the converse inclusion holds since $R$ is a Noetherian domain and $P_{\alpha}$ is a $m$-primary ideal for all $\alpha$.

Remark. Notice that for type E valuations, it is also true that the set of cosets in $P_{\nu\left(q_{i}\right)} / P_{\nu\left(q_{i}\right)^{+}}$of a minimal generating sequence $\left\{q_{i}\right\}_{i \in I}$ spans the $k$-algebra $\operatorname{gr}_{\nu} R$. 
On the other hand, associated with an order function $o: T \rightarrow \Gamma_{-\infty}$, one can also define its graded algebra as $\mathcal{G}:=\bigoplus_{\alpha \in \Gamma} O_{\alpha} / O_{\alpha^{-}}$, where $O_{\alpha}:=\{f \in T \mid o(f) \leq \alpha\}$ and $O_{\alpha^{-}}:=\{f \in T \mid o(f)<\alpha\}$.

The following result collects the consequences in terms of order functions of the above developed theory. There, we shall consider an order function associated with a valuation $\nu$ with value semigroup $S$. In such a case, we slightly modify the definition of graded algebra: set $o=-\nu$ and suppose that $T$ is any $k$-algebra of $K$ such that $S \subseteq o(T)$, then the sets $O_{\alpha}, \alpha \in S$ are $k$-vector spaces and we define the graded algebra $\operatorname{gr}_{o} T$ as above but grading it in $S$, the value semigroup of $\nu$, i.e. $\operatorname{gr}_{o} T:=\bigoplus_{\alpha \in S} O_{\alpha} / O_{\alpha^{-}}$.

Theorem 4.1. Let $\nu$ be a valuation of the fraction field $K$ of a 2-dimensional Noetherian local regular domain $R$ which is centered at $R$. Assume that $\nu$ is of type B-II-a, C, D or $E$ and let $\left\{q_{i}\right\}_{i \in I}$ be a minimal generating sequence of $\nu$. Then

(1) The function o $(=-\nu)$ defined over the $k$-algebra $\operatorname{gr}_{o} T, T:=k\left[\left\{q_{i}^{-1}\right\}_{i \in I}\right] \subseteq K$, is a weight function whose value semigroup is $S$, the value semigroup of $\nu$.

(2) The graded algebra associated with $\nu$ (relative to $R$ ) and that associated with o are isomorphic and both are isomorphic to the k-algebra of the semigroup $S, k[S]$.

(3) Assume that $\nu$ is of type B-II-a, C or D. Then, any Noetherian k-algebra $T \subseteq K$ such that

(i) $o: \operatorname{gr}_{o} T \rightarrow S$ is a weight function,

(ii) there exist elements $f_{i} \in T(0 \leq i \leq s<\infty)$ such that $f_{i}^{-1} \in R$ and the set $\left\{o\left(f_{i}+O_{o\left(f_{i}\right)^{-}}\right)\right\}_{0 \leq i \leq s}$ spans $S$

must be of the above form, that is $T=k\left[\left\{q_{i}^{-1}\right\}_{i \in I}\right],\left\{q_{i}\right\}_{i \in I}$ being a minimal generating sequence of $\nu$.

(4) Parametric equations for the function o, which allow the explicit computation of $o(h), h \in K$ are those given in Section 3.3.

Proof. The same procedure of Proposition 2.2 proves that $\operatorname{dim} O_{\alpha} / O_{\alpha^{-}}=1, \alpha \in S$, and this proves (1). Clause (2) is a consequence of the following $k$-algebra isomorphisms $\operatorname{gr}_{o} T \cong k[S] \cong \operatorname{gr}_{\nu} R$, which hold by fixing elements of each value in $T$ or $R$. Pick a family $\left\{f_{i}\right\}_{0 \leq i \leq r \leq s}$ such that the sequence $\left\{o\left(f_{i}+O_{o\left(f_{i}\right)^{-}}\right)\right\}_{0 \leq i \leq r}$ is a minimal set of generators of the semigroup $S$. The structure of $S$ shows that $T=K\left[\left\{f_{i}\right\}_{0 \leq i \leq r}\right]$. Since the set $\left\{f_{i}^{-1}+P_{o\left(f_{i}\right)^{+}}\right\}_{0 \leq i \leq r}$ spans $\operatorname{gr}_{\nu} R$, we have completed the proof of (4) by Proposition 4.1. Finally, Clause $(\overline{4})$ is clear from Section 3.3, where we have seen how to compute $o(h)$ for any $h$.

\section{Evaluation COdes Associated With the SEMigroup AlgEBra}

Along this section $o: \operatorname{gr}_{o} T \rightarrow S$ denotes the weight function given by a plane valuation of type B-II-a, C, D or E and by a minimal generating sequence $\left\{q_{i}\right\}_{i \in I}$ of it. Recall that we have set $T=k\left[\left\{q_{i}^{-1}\right\}_{i \in I}\right]$ and $\operatorname{gr}_{o} T \cong K[S]$. Assume that $\phi: \operatorname{gr}_{o} T \rightarrow k^{n}$ is an epimorphism of $k$-algebras. From $\phi$ and $o$, one can define a family of evaluation codes $\left\{E_{\alpha}\right\}_{\alpha \in S}$ in the following way

$$
E_{\alpha}=\operatorname{span}_{k}\left\{\phi(f) \mid o(f) \leq \alpha ; f \in \operatorname{gr}_{o} T\right\} .
$$


The dual space of the vector space $E_{\alpha}$ will be denoted by $C_{\alpha}$. Since $C_{\alpha}=0$ for $\alpha$ large enough, we denote by $\omega$ the least element in $S$ satisfying $C_{\alpha}=0$ for all $\alpha \geq \omega$. Our aim is to give a bound for the minimum distance of the code $C_{\alpha}$. To do this, we shall study the semigroup $S$ which has the advantage that it is that of a plane valuation.

It is clear that the set the values $\left\{\bar{\beta}_{i}:=o\left(q_{i}^{-1}\right)=\nu\left(q_{i}\right)\right\}_{0 \leq i<r}$, said to be of maximal contact, where $r=g+2$ if $\nu$ is of type $\mathrm{B}, \mathrm{C}$ or $\mathrm{D}$, and $r=\infty$ when $\nu$ is of type $E$, is a minimal set of generators of the semigroup $S$.

There is no lose of generality if we assume that the group of values of $\nu$ is a subgroup of $\mathbb{Z}^{2}$ if $\nu$ is of type $\mathrm{B}$ or $\mathrm{C}$ and it is a subgroup of $\mathbb{R}$ in the remaining cases.

The values $\left\{\bar{\beta}_{i}\right\}_{0 \leq i<r}$ can be obtained from the Hamburger-Noether expansion of $\nu$. This is a consequence of the facts given in Section 3.3 and from those formulae given in [1] for germs of plane curves. Explicitly, set $\beta_{0}^{\prime}=1$ and for $1 \leq i<r$

$$
\beta_{i}^{\prime}:=\left(h_{s_{i-1}}-k_{i-1}+1\right)+\frac{1}{h_{s_{i-1}+1}+\cdots+\frac{1}{k_{i}^{+}}},
$$

where $k_{i}^{+}$means either the element $k_{i}$ or the remaining sums (which could be infinite) of the Hamburger-Noether expansion whenever $i=g+1$ and $\nu$ is of type $\mathrm{C}$ or D. Clearly $\beta_{i}^{\prime} \in \mathbb{Q}$ for $i<r-1, \beta_{g+1}^{\prime}=\infty$ in case $\mathrm{B}, \beta_{g+1}^{\prime} \in \mathbb{Q}$ in case $\mathrm{C}$ and $\beta_{g+1}^{\prime} \in \mathbb{R} \backslash \mathbb{Q}$ in case D. Then $\bar{\beta}_{0}=\nu(u)$, and for $0 \leq i<r-1$,

$$
\bar{\beta}_{i+1}=m_{i} \bar{\beta}_{i}+\left(\beta_{i+1}^{\prime}-1\right) e_{i},
$$

where $m_{i}$ is the denominator of $\beta_{i}^{\prime}$ when we write it as a quotient of relatively prime elements and $e_{i}=\nu\left(w_{s_{i}}\right)$. Notice that

$$
\left(\beta_{i+1}^{\prime}-1\right) e_{i}=e_{i}\left(h_{s_{i}}-k_{i}\right)+\nu\left(w_{s_{i}+1}\right) .
$$

Theorem 5.1. Let $\left\{C_{\alpha}\right\}_{\alpha \in S}$ be the family of dual codes of $\left\{E_{\alpha}\right\}_{\alpha \in S}$ defined by a weight function $o: \operatorname{gr}_{o} T\left(T:=k\left[\left\{q_{i}^{-1}\right\}_{0 \leq i<r}\right]\right) \rightarrow S$ which comes from a type $B-I I-a, C, D$ or $E$ plane valuation $\nu$ and a minimal generating sequence $\left\{q_{i}\right\}_{0 \leq i<r}$ of $i$, and $\phi: \operatorname{gr}_{o} T \rightarrow k^{n}$ an epimorphism of $k$-algebras. Set $\left\{\bar{\beta}_{i}\right\}_{0 \leq i<r}$ a minimal system of generators of the semigroup $S,\left\{e_{i}\right\}_{0 \leq i<r-1}$ the family of values $e_{i}=\nu\left(w_{s_{i}}\right)$ and $n_{i}$ those positive integers such that $e_{i} n_{i}=e_{i-1}$.

Then, a bound for the minimum distance of the code $C_{\alpha}$ is

$$
\min \left[\prod_{i=0}^{d}\left(a_{i}+1\right)\right]-2,
$$

where $\left(a_{0}, a_{1}, \ldots, a_{d}\right)$ runs over the unique coefficients of the expressions of those elements $\delta \in S(\omega>\delta>\alpha)$ of the form $\delta=\sum_{i=0}^{d} a_{i} \bar{\beta}_{i}\left(a_{i} \in \mathbb{N}\right), d=r-1$ and $a_{i}<n_{i}(1 \leq i<d)$ if $\nu$ is not of type $E$, and $d<r$ and $a_{i}<n_{i}(1 \leq i \leq d)$ otherwise.

Proof. Recall that if $\nu$ is of type $\mathrm{B}, \bar{\beta}_{r}=(1,0)$ (and the first coordinate of the remaining values $\bar{\beta}_{i}$ is 0 ), when $\nu$ is of type $\mathrm{C}$, then $\bar{\beta}_{r}$ does not belong to the line joining the origin to $\bar{\beta}_{0}$, unlike the other values $\bar{\beta}_{i}$, and in the case of type $\mathrm{D}$ valuations, one gets $\bar{\beta}_{r} \in \mathbb{R} \backslash \mathbb{Q}$, but the remaining values $\bar{\beta}_{i}$ are rational numbers. Therefore, for fixed $\delta \in S$, the coefficient $a_{r}$ of any expression $\delta=\sum_{i=0}^{r} a_{i} \bar{\beta}_{i}$ for the above types of valuations is unique. 
Furthermore, assume that

$$
\sum_{i=0}^{r-1} a_{i} \bar{\beta}_{i}=\sum_{i=0}^{r-1} a_{i}^{\prime} \bar{\beta}_{i}
$$

and $a_{i}\left(a_{i}^{\prime}\right.$, respectively) $<n_{i}(1 \leq i<r)$. Then, it is clear that $a_{r-1}^{\prime}-a_{r-1}$ is a multiple of $e_{r-1}$ and so it is a multiple of $n_{r-1}$, which contradicts $a_{r-1}<n_{r-1}$. Repeating this procedure for $r-2, r-3, \ldots$, one leads to the uniqueness of the above expression. Notice that this last reasoning is also valid for valuations of type E.

Finally, a bound for the minimum distance follows in an straightforward way from the uniqueness of the above expression of $\delta$ and from the fact that a bound for the minimum distance of the code $C_{\alpha}$ is given by

$$
\min \left\{\mu_{\delta} \mid \delta \in S, \delta>\alpha \text { and } C_{\delta} \neq C_{\delta^{+}}\right\},
$$

$\mu_{\delta}$ being the cardinality of the set $\left\{\left(\delta_{1}, \delta_{2}\right) \in S^{2} \mid \delta_{1}+\delta_{2}=\delta\right\}$ and $\delta^{+}$the minimum of the set $\{\gamma \in S \mid \gamma>\delta\}$. This fact can be proved following the line given in [8] for subsemigroups of the semigroup $\mathbb{N}$ of the nonnegative integers.

Remark. Valuations of type $\mathrm{E}$ are interesting from the point of view of order functions. As we shall see, they do not give monomial orderings. Note that the example of [9] cited in the introduction corresponds to this type of valuations. To handle these valuations is not easy because we can obtain their parametric equations only in characteristic zero and, even in this case, we get power series with infinitely many data which are not suitable for an algorithm. However, we shall handle many type $\mathrm{E}$ valuations by using the fact that they can be regarded as a limit of type A ones.

For that reason, although we have yet mentioned this fact, now we shall be more precise. Let $\pi$ the sequence (1) of a type E valuation $\nu$. Consider the finite subsequence of $\pi$

$$
X_{i} \stackrel{\pi_{i}}{\longrightarrow} X_{i-1} \longrightarrow \cdots \longrightarrow X_{1} \stackrel{\pi_{1}}{\longrightarrow} X_{0}=X=\operatorname{Spec} R,
$$

and the corresponding valuation $\nu_{i}$ (of type A). Then, for every $f \in K$, there exists a nonnegative integer $m(f)$, that depends on $f$, such that $\nu(f)=\nu_{i}(f)$ whenever $i>m(f)$. We have assumed that all valuations are normalized in such a way that the minimum value by $\nu$ or $\nu_{i}$ of the elements in the maximal ideal of $R$ is 1 .

The proof of the above fact follows after realizing that the map $\nu *$, defined on $K$ as $\nu^{*}(f)=\lim _{i \rightarrow \infty} \nu_{i}(f)$, is a valuation of $K$ which assigns to $f$ the value $\nu_{i}(f)$ for $i$ large enough, and that $\nu$ and $\nu *$ are equivalent valuations.

To see the first assertion, it suffices to consider $f \in R$, analytically irreducible, and note that if we consider divisorial valuations $\nu_{i}, i \geq i_{0}$, whose Hamburger-Noether expansion is large enough to have the same common part with the Hamburger-Noether expansion of the germ of plane curve given by $f$ (in the corresponding basis of the maximal ideal of $R$ and $R /(f) R$ ), then $\nu_{i}(f)$ have the same value for all $i \geq i_{0}$ (use (3) and the known theory of plane algebroid curves [1]). Finally, by noticing that the valuation ring $R_{\nu}$ is the directed limit $\lim _{\rightarrow} \mathcal{O}_{X_{i}, P_{i}}$, that $R_{\nu_{i}}=\mathcal{O}_{X_{i}, \eta_{i}}$, for $\eta_{i}$ the generic point of the defining divisor of $\nu_{i}$, and that there exists a one to one morphism of local rings $\mathcal{O}_{X_{i}, P_{i}} \rightarrow \mathcal{O}_{X_{i+1}, \eta_{i+1}}$ we conclude $R_{\nu}=R_{\nu^{*}}$. 
The following result allows us to regard $\operatorname{gr}_{\nu} R$ as a quotient of a polynomial algebra. Since $\operatorname{gr}_{o} T \cong \operatorname{gr}_{\nu} R \cong k[S]$, this completes the construction of our codes.

Theorem 5.2. Let $\nu$ be a plane valuation of type B-II-a, C, D or E, $\left\{\bar{\beta}_{i}\right\}_{0 \leq i<r}$ the generators of the value semigroup of $\nu$ and $\left\{q_{i}\right\}_{0 \leq i<r}$ a minimal generating sequence of $\nu$. Set $A[\nu]=k\left[\left\{X_{i}\right\}_{0 \leq i<r}\right]$ and define $\psi: A[\nu] \rightarrow g r_{\nu} R$, by $\psi\left(X_{i}\right)=q_{i}+P_{\nu\left(q_{i}\right)^{+}} \cdot \psi$ is an epimorphism of $k$-algebras. Consider, for each $i<r-1$, the unique expression

$$
n_{i} \bar{\beta}_{i}=\sum_{j=0}^{i-1} \gamma_{i j} \bar{\beta}_{j}
$$

where $\gamma_{i j}$ are nonnegative integers such that $\gamma_{i j}<n_{j}$ and $n_{j}(j \leq i)$ the values defined in Theorem 5.1. If $a_{i} \in k$ is that value satisfying

$$
q_{i}^{n_{i}}+P_{\left(n_{i} \bar{\beta}_{i}\right)^{+}}=a_{i}\left(\prod_{j=0}^{i-1} q_{j}^{\gamma_{i j}}+P_{\left(n_{i} \bar{\beta}_{i}\right)^{+}}\right),
$$

then the ideal of $A[\nu]$, $\operatorname{ker} \psi$, is spanned by the set $g_{i}:=a_{i} X_{i}^{n_{i}}-\prod_{j=0}^{i-1} X_{j}^{\gamma_{i j}},(0 \leq i<r-1)$.

Proof. Consider the subset $G$ of $A[\nu]$ whose elements are expressions $w=a \pi_{1}+b \pi_{2}$ such that $\pi_{l}(l=1,2)$ are forms with coefficient 1 that satisfy $\nu\left(\bar{\pi}_{1}\right)=\nu\left(\bar{\pi}_{2}\right)$ and $\nu\left(a \overline{\pi_{1}^{\prime}}+\right.$ $b \nu\left(\bar{\pi}_{1}^{\prime}\right)>\nu\left(\overline{\pi_{1}^{\prime}}\right)$, where $\bar{\pi}_{l}$ means substituting $X_{i}$ by $q_{i}$ in $\pi$ and where $\pi_{l}^{\prime}$ equals $\pi_{l} / X_{r-1}^{b}$ if $\nu$ is of type B and $b$ is the exponent of $X_{r-1}$ in $\pi_{l}$, and $\pi_{l}^{\prime}=\pi_{l}$ otherwise. $G$ generates ker $\psi$.

If $\nu$ is not of type $\mathrm{E}$, each expression $w$, up to constant, can be represented by a pair $(\alpha, \beta) \in \mathbb{N}^{r} \times \mathbb{N}^{r}$ where $\alpha$ and $\beta$ are the ordered exponents of the $X_{i}$ in $\pi_{1}$ and $\pi_{2}$. The obtained set $\Omega$ is a congruence (i.e., an equivalence relation on $\mathbb{N}^{r}$ such that $(\alpha+\gamma, \beta+\gamma) \in \Omega$ whenever $(\alpha, \beta) \in \Omega$ and $\left.\gamma \in \mathbb{N}^{r}\right)$. Now, if $\Delta$ is the subset of $\Omega$ consisting of the pairs representing the elements in the set $\left\{g_{i}\right\}_{0 \leq i<r-1}$, we conclude the result after noticing that $\Omega$ is the smallest congruence containing $\Delta$. We arrive at that conclusion by getting an element in $\Delta$ from any element in $\Omega$ by repeating, for each coordinate $z$ of $\alpha$ and $\beta$, the following procedure: if $z-n_{i} \bar{\beta}_{i}>0$ ( $i$ as large as possible), we replace $(\alpha, \beta)$ by another element $\left(\alpha^{\prime}, \beta^{\prime}\right)$ where we have subtracted $n_{i}$ to the $i$ th coordinate of $\alpha$ or $\beta$ (what corresponds to $z$ ) and we have added $\gamma_{i j}$ to the $j$ th one according to the expression (7).

The above reasoning with minor changes proves the same property for type A valuations. In the above remark, we have shown that if $\nu$ is a valuation of type E, we can consider a sequence of valuations of type A, $\left\{\nu_{i}\right\}_{i \geq 0}$, converging to $\nu$. It is clear that picking a large enough positive integer $i_{0}$, then the (normalized) values of $\nu_{i}, i \geq i_{0},\left\{e_{i}, \bar{\beta}_{i}\right\}_{i \leq g}$ (which can be computed as above, $r=g+1$ ) are the same as the $g$ first of $\nu$. Thus, if $h \in \operatorname{ker} \psi, h$ will be in the ideal $\left\langle g_{i}\right\rangle_{i \leq i_{0}}$ for some $i_{0}$.

Remark. Notice that we can change the element $q_{i}$ of the minimal generating sequence by $a_{i}^{1 / n_{i}} q_{i}$. In this case $g_{i}=X_{i}^{n_{i}}-\prod_{j=0}^{i-1} X_{j}^{\gamma_{i j}},(0 \leq i<r-1)$. 


\section{Other weight functions. Algorithms And examples}

Proposition 2.2 and 2 of Theorem 4.1 give a computable weight function for each suitable plane valuation and $k$-algebra. The next straightforward result provides some ordered domains suited to the above results.

Proposition 6.1. Consider a valuation $\nu$ and notations as in Theorem 5.2. 1. If $\nu$ is of type $B, C$ or $D$ and $q \in k\left[q_{0}^{-1}, q_{1}^{-1}, \ldots, q_{r-2}^{-1}\right]$ such that $o(q)>0$, then:

a. The map o: $k\left[q, q_{r-1}^{-1}\right] \rightarrow o\left(k\left[q, q_{r-1}^{-1}\right]\right)$ is a weight function.

b. The map o : $k\left[q^{-a} q_{r-1}^{-b}, q^{-c} q_{r-1}^{-d}\right] \rightarrow o\left(k\left[q^{-a} q_{r-1}^{-b}, q^{-c} q_{r-1}^{-d}\right]\right)$ is a weight function whenever $\nu$ is of type $B$ and $a \nu(q)+b \nu\left(q_{r-1}\right) \neq c \nu(q)+d \nu\left(q_{r-1}\right)$ for $a, b, c, d$ positive integers.

2. For $\alpha \in S$ such that $\alpha<n_{1} \bar{\beta}_{1}$, set $\Pi_{\alpha}$ the unique monic monomial in $q_{0}$ and $q_{1}$ whose valuation is $\alpha$, and

$$
\Delta_{1}:=\frac{1}{q_{1}^{n_{1}}}-a_{1} \frac{1}{q_{0}^{\gamma_{10}}} .
$$

When $o\left(\Delta_{i-1}\right) \in S(i>1)$, define inductively $\Delta_{i}:=\Delta_{i-1}-\lambda_{i} \Pi_{o\left(\Delta_{i-1}\right)}^{-1}$, $\lambda_{i}$ being the unique element in $k$ such that $o\left(\Delta_{i}\right)<o\left(\Delta_{i-1}\right)$. If $o\left(\Delta_{i_{0}}\right)>0$ and $o\left(\Delta_{i_{0}}\right) \notin S$ for some $i_{0} \geq 1$, then $o: k\left[q_{0}^{-1}, q_{1}^{-1}\right] \rightarrow o\left(k\left[q_{0}^{-1}, q_{1}^{-1}\right]\right)$ is a weight function, and

$$
o: k\left[q_{0}^{-1}, q_{1}^{-1}, q_{r-1}^{-1}\right] \rightarrow o\left(k\left[q_{0}^{-1}, q_{1}^{-1}, q_{r-1}^{-1}\right]\right)
$$

is also whenever $\nu$ is not of type $E$.

Remark. We have explained how to construct codes and parametric equations for weight functions coming from plane valuations. Next, we shall clarify why both constructions are algorithmic. The algorithm for constructing the semigroup algebra $K[S]$ is named A1 and A2 that for obtaining the parametric equations and some suitable ordered domains. A2 also allows us to get representatives of every class in $\operatorname{gr}_{o} T$. A1 is very simple and A2 is supported on other algorithms relative to curve singularities. The parametric equations of algorithm A2 will allow us to explicitly compute the order functions of the above Proposition. The last row of the Hamburger-Noether expansions of valuations of type B can be infinite, however we can consider the so-called symbolic Hamburger-Noether expansion for them (see [2]). This expansion contains as a last row an implicit equation in $w_{s_{g}}$ and $w_{s_{g-1}}$, which allows us to explicitly obtain as many elements of this last row as we want. So we can use this symbolic expansion to compute the valuation $\nu$ of any element of the field as we have described by successive substitution and lazy evaluation.

The input of our algorithm $\mathbf{A} 1$ is a convenient form of the Hamburger-Noether expansion of a plane valuation $\nu$ (types B-II-a, C, D or E). For valuations of type B, we only need to know the first element in the last row of their expansion, which can be expressed as $w_{s_{g}-1}=a_{s_{g} k_{g}} w_{s_{g}}^{k_{g}}+$. As we have said, if $\nu$ is of type C, we must also fix two pairs in $\mathbb{Z}^{2},(0, b)$ and $(c, d)$ corresponding to $\nu\left(w_{z}\right)$ and $\nu\left(w_{z-1}\right)$. For type $\mathrm{D}$ valuations, we change the last infinite set of non-free rows $w_{i-1}=w_{i}^{h_{i}} w_{i+1},\left(i>s_{g}\right)$ by an expression $w_{s_{g}}=w_{s_{g-1}}^{\delta}$, where, as above, $\delta$ is the real, non rational, number given by the continued fraction $\left[h_{s_{g}+1} ; h_{s_{g}+2}, h_{s_{g}+3}, \ldots\right]$. Finally, we can obtain the Hamburger-Noether expansion of infinitely many valuations of type E simply by giving some recursive procedure 
which provides the values $a_{i j}, h_{j}, k_{j}$ and $s_{i}$.

The input of our algorithm A2 is what we call the symbolic Hamburger-Noether expansion of a plane valuation $\nu$. When $\nu$ is a valuation of type $\mathrm{B}$, we consider an algebraic curve given by $f \in R$ such that it is analytically irreducible and compute its symbolic Hamburger-Noether expansion as in [2] which will be the same of $\nu$. That algorithm allows us to decide if a polynomial corresponds to an analytically irreducible curve. To compute implicit equations of some generating sequence of $\nu$ we can use lazy evaluation for polynomials from the parametric equations that the symbolic Hamburger-Noether expansion gives or to use the algorithm given in [3] to that purpose. Notice that $\nu$ corresponds to a curve given by a polynomial. For valuations $\nu$ of types $\mathrm{C}$ and $\mathrm{D}$, we can consider the symbolic Hamburger-Noether expansion of a type $\mathrm{B}$ valuation $\nu^{*}$ and replace its last row by another set of rows that make the expansion into a type $\mathrm{C}$ or $\mathrm{D}$ valuation (the first new row corresponds partly to the last one of $\nu^{*}$ and the following ones can be chosen as we desire). This choice has the advantage that a generating sequence for $\nu$ is the same we know for $\nu^{*}$. Summing up, good choices of the Hamburger-Noether expansions give minimal generating sequences which are polynomial. Finally, for type E valuations, we can do the same, but we only know a part of the generating sequence.

Computation of A1 Reproducing the computations given in Section 5 (recall that $\beta_{g+1}^{\prime}=\left(h_{s_{g}}-k_{g}+1\right)+1 / \delta$ for type $\mathrm{D}$ valuations) and taking into account that the Hamburger-Noether expansion allows us to compute the values $e_{i}$ (since we know $\nu\left(w_{s_{g}}\right)$ ), we compute the values $\bar{\beta}_{i}$ and $n_{i}$ for all valuations but those of type $\mathrm{E}$. If $\nu$ is a valuation of type $\mathrm{E}$, by using the recurrence relations that give the Hamburger-Noether expansion, we can compute values $\bar{\beta}_{i}$ and $n_{i}$ relative to $\nu$ for $i \leq g_{0}, g_{0}$ as large as we want. To do it, we only need to reproduce the above computations with the portion of Hamburger-Noether expansion we need (what corresponds to some type A valuation of the set of those converging to $\nu)$ to get values $\bar{\beta}_{i}^{*}$ and $e_{i}\left(0 \leq i \leq g_{0}\right)$. Finally, the maximal contact values of $\nu$ will be $\bar{\beta}_{i}=\bar{\beta}_{i}^{*} / \bar{\beta}_{0}^{*}$.

Output of A1. The ordered domain $\operatorname{gr}_{o} T$ will be isomorphic to

$$
k\left[X_{0}, X_{1}, \ldots, X_{r-1}\right] /\left\langle g_{i} \mid 1 \leq i<r-1\right\rangle .
$$

Let us note that to obtain the order function, we only need to assign to each $X_{i}$ the weight $\bar{\beta}_{i}$ and the order of any element $f+\left\langle\left\{g_{i}\right\}\right\rangle$ will be the degree of the polynomial $f$ with respect to the assigned weight. If $\nu$ is a type $\mathrm{E}$ valuation and $f$ involves $l$ indeterminates, we only need to compute values $\bar{\beta}_{i}$ and $e_{i}(i \leq l)$ to get its order. Clearly this order is not monomial.

Output of A2. Parametric equations of the valuation. We have already said how to compute these equations from our input. This allows us to get $o(f)$ for any element $f \in K$. Recall that we know implicit equations for generating sequences of the valuations which allows us to know the ordered domain in Proposition 6.1.

Notice that for type E valuations, $\nu$, we can compute $o(f / g)$ where $f$ and $g$ are reduced elements in $R$ giving algebraic curves. To do it, we must compute the Hamburger-Noether expansion of the branches of $f$ and $g$ (the algorithm in [2] computes it) and choose the Hamburger-Noether expansion of a divisorial valuation $\nu_{i}$ converging to $\nu$ (gotten by a 
piece of the Hamburger-Noether expansion of $\nu$ ) which have the largest coincidence in values $a_{i j}, h_{j}, k_{j}, s_{i}$ to the branches of $f$ and $g$ (always using the same basis). Then $o(f / g)=-\nu(f / g)=-\bar{\nu}_{i}(f / g), \bar{\nu}_{i}$ being the normalization of the valuation $\nu_{i}$. Finally, $\nu_{i}(f / g)$ can be computed by using the parametric equations given in Section 3.3 for type A valuations.

\section{Examples.}

1. Consider the type B plane valuation $\nu$ of the field $k(u, v)$ centered at the local ring $k[u, v]_{(u, v)}$ whose Hamburger-Noether expansion in the regular system of parameters $\{u, v\}$ (input) is

$$
\begin{aligned}
v & =u w_{1} \\
u & =w_{1}^{2}+w_{1}^{2} w_{2} \\
w_{1} & =w_{2}^{2} .
\end{aligned}
$$

Notice that this is the same Hamburger-Noether expansion (for a suitable basis) of the curve of equation $f=0, f=v^{5}-u^{7}$. We can get $q_{0}=u, q_{1}=v, q_{2}=f$ and $\bar{\beta}_{0}=$ $(0,2), \bar{\beta}_{1}=(0,3), \bar{\beta}_{2}=(1,0), n_{1}=2$. So an output is $\operatorname{gr}_{o} T \cong k\left[X_{0}, X_{1}, X_{2}\right] /\left\langle X_{1}^{2}-X_{0}^{3}\right\rangle$. Given weight $(0,2)$ to $X_{0},(0,3)$ to $X_{1}$ and $(1,0)$ to $X_{2}$, we get the order function. Finally the parametric equations are $u=t^{4}+t^{5}, v=t^{6}+t^{7}$.

2. Similarly, if we consider a type $\mathrm{C}$ plane valuation whose input is

$$
\begin{aligned}
v & =u w_{1} \\
u & =w_{1}^{2}+w_{1}^{2} w_{2} \\
w_{1} & =w_{2}^{\pi} .
\end{aligned}
$$

we get $q_{0}=u, q_{1}=v, q_{2}=v^{3}-u^{2}$ and computing $\bar{\beta}_{0}=2, \bar{\beta}_{1}=3, \bar{\beta}_{2}=6+\frac{1}{\pi}, n_{1}=2$. As above an output is $\operatorname{gr}_{o} T \cong k\left[X_{0}, X_{1}, X_{2}\right] /\left\langle X_{1}^{2}-X_{0}^{3}\right\rangle$, but we must give weight $6+\frac{1}{\pi}$ to the indeterminate $X_{2}$. The parametric equations are $u=t^{2 \pi}+t^{2 \pi+1}, v=t^{3 \pi}+t^{3 \pi+1}$.

3. Let $\nu$ be a type $\mathrm{E}$ valuation whose input is

$$
\begin{aligned}
v & =u w_{1} \\
u & =w_{1}^{2}+w_{1}^{2} w_{2} \\
w_{1} & =w_{2} w_{3} \\
w_{2} & =w_{3}^{2}+w_{3}^{2} w_{4} \\
\cdots & \cdots
\end{aligned}
$$

That is, the Hamburger-Noether expansion repeats indefinitely the structure of the two first rows. The two first rows give $\bar{\beta}_{0}^{*}=2, \bar{\beta}_{1}^{*}=3, n_{1}=2$. If we consider two more ones, then $\bar{\beta}_{0}^{*}=4, \bar{\beta}_{1}^{*}=6, \bar{\beta}_{3}^{*}=7, n_{1}=2, n_{2}=2$. So we get $\bar{\beta}_{0}=1, \bar{\beta}_{1}=3 / 2, \bar{\beta}_{3}=$ $7 / 4, \ldots, n_{1}=2, n_{2}=2, \ldots$ and $\operatorname{gr}_{o} T \cong k\left[X_{0}, X_{1}, X_{2}, \ldots\right] /\left\langle X_{1}^{2}-X_{0}^{3}, X_{2}^{2}-X_{0}^{2} X_{1}, \ldots\right\rangle$.

4. Let us see a more complicated example. For simplicity, we consider zero characteristic. The input is

$$
\begin{array}{ccc}
v & = & u w_{1} \\
u & = & -w_{1}^{2}+w_{1}^{2} w_{2} \\
w_{1} & = & -w_{2}^{2}+w_{2}^{2} w_{3} \\
w_{2} & = & -w_{3}^{2}+w_{3}^{2} w_{4} \\
w_{3} & = & w_{4}^{\sqrt{2}},
\end{array}
$$


then we get $q_{0}=u, q_{1}=v, q_{2}=u^{3}+v^{2}, q_{3}=u^{6}+2 u^{3} v^{2}+v^{4}+v u^{5}, q_{4}=v^{8}+4 v^{6} u^{3}+$ $2 v^{5} u^{5}+6 v^{4} u^{6}+4 v^{3} u^{8}+2 v^{2} u^{9}(u+2)+2 v u^{11}+u^{12}(u+1)$ and computing $\bar{\beta}_{0}=16, \bar{\beta}_{1}=$ $24, \bar{\beta}_{2}=52, \bar{\beta}_{3}=106, \bar{\beta}_{4}=(424+\sqrt{2}) / 2, n_{1}=n_{2}=n_{3}=2$. An output is $\operatorname{gr}_{o} T \cong$ $k\left[X_{0}, X_{1}, X_{2}, X_{3}, X_{4}\right] /\left\langle X_{1}^{2}-X_{0}^{3}, X_{2}^{2}-X_{0}^{2} X_{1}^{3}, X_{3}^{2}-X_{0}^{2} X_{1} X_{2}^{3}\right\rangle$. The parametric equations are $u=t^{16}(-1+t)^{4}\left(-1+t^{\sqrt{2}}\right)^{2}\left[-1+t^{2 \sqrt{2}}(1+t)\right], v=t^{24}(-1+t)^{6}\left(-1+t^{\sqrt{2}}\right)^{3}\left[-1+t^{2 \sqrt{2}}(1+t)\right]$. We have used SINGULAR and the algorithm in [3].

Our procedure works for the above described $k$-algebras $T$ whose transcendence degree (that of their quotient field) is two. Since the associated codes are given by order functions, we can use the Berlekamp-Massey-Sakata algorithm to decode them. To end this paper, we shall show that evaluation codes associated with order functions can be given for any finitely generated $k$-algebra (of arbitrary transcendence degree). The concrete result is the following:

Proposition 6.2. Let $J=\left\langle p_{j}\right\rangle(1 \leq j \leq s)$ be an ideal of the polynomial ring $k[X]:=$ $k\left[X_{1}, \ldots, X_{m}\right]$. Consider a set $P=\left\{P_{1}, P_{2}, \ldots, P_{n}\right\}$ of points in the zero set of $J$. Set $\operatorname{ev}_{P}: k[X] / J \rightarrow k^{n}$ the evaluation map given by $\operatorname{ev}_{P}(h+J)=\left(h\left(P_{1}\right), h\left(P_{2}\right), \ldots, h\left(P_{n}\right)\right)$. Then, there exists a family of vector spaces $\left\{W_{\alpha}\right\}_{\alpha \in S}$, where $S$ is a semigroup such that the family of codes $\left\{E_{\alpha}:=\operatorname{ev}_{P}\left(W_{\alpha}\right)\right\}_{\alpha \in S}$ comes from an order function over a suitable $k$-algebra, and so the dual codes can be quickly decoded by the Berlekamp-Massey-Sakata algorithm.

Proof. On polynomial ring $k[X]$, consider weights $w\left(X_{i}\right) \in \mathbb{N}^{r}(1 \leq i \leq m)$ which allow us to give a weight, in the obvious manner, to each element of the set $M$ of monomials in $k[X]$. If, in addition, we consider a monomial ordering $<$ on $\mathbb{N}^{r}$ and another one $<_{M}$ on $M$, then we get a generalized weighted degree ordering on $M$ associated with $<_{M}$ and $<$, denoted $<_{w}$, so: $M_{1}<_{w} M_{2}$ where $M_{1}, M_{2} \in M$ if, and only if, either $w\left(M_{1}\right)<w\left(M_{2}\right)$ or $w\left(M_{1}\right)=w\left(M_{2}\right)$ and $M_{1}<_{M} M_{2}$.

Take the polynomial ring $k\left[X_{1}, \ldots, X_{m}, U_{1}, \ldots, U_{s}\right]:=k[X, U]$, pick linearly independent weights $w\left(X_{i}\right)$ and give to each $U_{j}$ the highest weight of the monomials in $p_{j}$ $(1 \leq j \leq s)$. Consider a monomial ordering on the set $M$ of monomials in $k[X, U]$ such that $U_{j}>X_{i}$ for all $j$ and $i$. Now, consider the ideal $I$ of $k[X, U]$ spanned by $p_{j}+U_{j}$ $(1 \leq j \leq s)$. Then, a weight function $o: k[X, U] / I \rightarrow S_{\infty}$ exists. Indeed, to show this, we are going to check the three facts which allow to apply the factor ring Theorem of [6].

Firstly, we note that the $S$ polynomial $S_{j l}$ (see [4, Ch. II, Sect. 6, Def. 4]) of the polynomials $p_{j}$ and $p_{l}$ is $-p_{l} U_{j}+p_{j} U_{l}, j, l \in\{1,2, \ldots, s\} ; j \neq l$ and thus the remainder of dividing $S_{j l}$ by $p_{j}+U_{j}$ and $p_{l}+U_{l}$ is equal to 0 , and so [4, Ch. II, Sect. 6, Th. 6] we have proved that the set $G=\left\{p_{j}+U_{j}\right\}_{1 \leq j \leq s}$ is a Gröbner basis for $I$.

Secondly, we notice that the set

$$
\Delta_{<w}=\left\{M^{\prime} \in M \mid M^{\prime} \text { is not a leading monomial of any monomial in } I\right\},
$$

called the footprint of $I$ is the set of monomials in $k[X]$ and they have mutually distinct weights since the weights $w\left(X_{i}\right)$ are linearly independent. 
Finally, by construction, every polynomial $p_{j}+U_{j}$ has, exactly, two monomials of highest weight in its support. So, we obtain a weight function $o: k[X, U] / I \rightarrow S_{-\infty}$ given by

$$
o(f)=\max _{<}\left\{w\left(M^{\prime}\right) \mid M^{\prime} \in \operatorname{Supp}(F)\right\}
$$

$F$ being the remainder on division by $G$ of any polynomial in $f$, whenever $f \neq 0$, and otherwise by $o(f)=-\infty$.

Now, the kernel of the epimorphism of rings $\varphi: k[X, U] \rightarrow k[X] / J$, given by $\varphi(h(X, U))=$ $h(X, 0)+J$ is the ideal $L$ of $k[X, U]$ spanned by the set $\left\{p_{j}, U_{j}\right\}_{1 \leq j \leq s}$. Thus, one obtains an isomorphism $k[X, U] / L \cong k[X] / J$. On the other hand, we can consider the natural ring epimorphism $\mu: k[X, U] / I \rightarrow k[X, U] / L$ which holds since $I \subseteq L$. Set $i: k^{m} \rightarrow k^{m+s}, i\left(x_{1}, \ldots, x_{m}\right)=\left(x_{1}, \ldots, x_{m}, 0, \ldots, 0\right)$ and consider the set of points $Q=\left\{Q_{i}=i\left(P_{i}\right)\right\}_{1 \leq i \leq n}$. The map $\operatorname{ev}_{Q}: k[X, U] / I \rightarrow k^{n}$ is a surjective morphism of $k$ algebras [8]. Therefore, for each $\alpha \in S$, we can consider the vector subspaces of $k[X, U] / I$

$$
W_{\alpha}=\operatorname{span}_{k}\{f \in k[X, U] / I \mid o(f) \leq \alpha\},
$$

and it is clear that the set of codes $\left\{E_{\alpha}\right\}_{\alpha \in S}$, where $E_{\alpha}=\operatorname{ev}_{Q}\left(W_{\alpha}\right)$, is a family of evaluation codes associated with a Noetherian order domain. Finally, it is straightforward that if we write $V_{\alpha}=\mu\left(W_{\alpha}\right)$, then the family $\left\{E_{\alpha}\right\}_{\alpha \in S}$ is exactly $\left\{\operatorname{ev}_{P}\left(V_{\alpha}\right)\right\}_{\alpha \in S}$ which concludes the proof.

\section{REFERENCES}

[1] A. Campillo, "Algebroid curves in positive characteristic", Lecture Notes in Math. 613. SpringerVerlag (1980).

[2] A. Campillo and J.I. Farrán, Symbolic Hamburger-Noether expressions of plane curves and applications to AG codes, Math. Comput. 71 (240) (2002), 1759-1780.

[3] V. Cossart and G. Moreno-Socías, Abhyankar's irreducibility criterion: a geometric point of view. Preprint University of Versailles.

[4] D. Cox, J. Little and D. O'Shea "Ideals, varieties and algorithms", Springer (1996).

[5] F. Delgado, C. Galindo and A. Nuñez, Saturation for valuations on two-dimensional regular local rings, Math. Z. 234 (2000), 519-550.

[6] O. Geil and R. Pellikaan, On the structure of order domains, Finite Fields Appl. 8 (2002), 369-396.

[7] G.M. Greuel, G. Pfister and H. Schoenemann, SINGULAR, a computer algebra system for Commutative Algebra and Algebraic Geometry. Fachbereich Mathematik der Universität, Kaiserlautern.

[8] T. Hoholdt, J.H. van Lint and R. Pellikaan, "Algebraic geometry codes" in Handbook of coding theory, Vol. 1, (1998), 871-961.

[9] M. E. O'Sullivan, New codes for the Belekamp-Massey-Sakata algorithm, Finite Fields Appl. 7 (2001), 293-317.

[10] M. Spivakovsky, Valuations in function fields of surfaces, Amer. J. Math. 112 (1990), 107-156.

[11] O. Zariski, The reduction of singularities of an algebraic surface, Ann. Math. 40 (1939), 639-689.

[12] O. Zariski and P. Samuel, "Commutative Algebra. vol II", Springer-Verlag (1960).

Current address: Departament de Matemàtiques, Universitat Jaume I, Campus de Riu Sec. s/n, 8029 A.P. Castelló (Spain)

E-mail address: galindo@mat.uji.es sanchis@mat.uji.es 